Published in final edited form as:

Ann Pharmacother. 2018 August ; 52(8): 713-723. doi:10.1177/1060028018759343.

\title{
Critical Care Pharmacists and Medication Management in an ICU Recovery Center
}

\author{
Joanna L. Stollings, PharmD, FCCM, FCCP ${ }^{1}$, Sarah L. Bloom, AGACNP-BC ${ }^{1}$, Li Wang, MS ${ }^{1}$, \\ E. Wesley Ely, MD, MPH, FCCM ${ }^{1,2}$, James C. Jackson, PsyD ${ }^{2,3}$, and Carla M. Sevin, MD ${ }^{1}$ \\ ${ }^{1}$ Vanderbilt University Medical Center, Nashville, TN, USA \\ ${ }^{2}$ Department of Veterans Affairs Medical Center Tennessee Valley Healthcare System, Nashville, \\ TN, USA
}

${ }^{3}$ Vanderbilt University School of Medicine, Nashville, TN, USA

\section{Abstract}

Background-Many patients experience complications following critical illness; these are now widely referred to as post-intensive care syndrome (PICS). An interprofessional intensive care unit (ICU) recovery center (ICU-RC), also known as a PICS clinic, is one potential approach to promoting patient and family recovery following critical illness.

Objectives-To describe the role of an ICU-RC critical care pharmacist in identifying and treating medication-related problems among ICU survivors.

\begin{abstract}
Methods-A prospective, observational cohort study was conducted of all outpatient appointments of a tertiary care hospital's ICU-RC between July 2012 and December 2015. The pharmacist completed a full medication review, including medication reconciliation, interview, counseling, and resultant interventions, during the ICU-RC appointment.
\end{abstract}

\begin{abstract}
Results-Data from all completed ICU-RC visits were analyzed $(n=62)$. A full medication review was performed in $56(90 \%)$ of these patients by the pharmacist. The median number of pharmacy interventions per patient was 4 (interquartile range $=2,5$ ). All 56 patients had at least 1 pharmacy intervention; 22 (39\%) patients had medication(s) stopped at the clinic appointment, and $18(32 \%)$ patients had new medication(s) started. The pharmacist identified $9(16 \%)$ patients who had an adverse drug event (ADE); 18 (32\%) patients had ADE preventive measures instituted. An influenza vaccination was administered to 13 (23\%) patients despite an inpatient protocol to ensure influenza vaccination prior to discharge. A pneumococcal vaccination was administered to $2(4 \%)$ patients.
\end{abstract}

\footnotetext{
Reprints and permissions: sagepub.com/journalsPermissions.nav

Corresponding Author: Joanna L. Stollings, Department of Pharmaceutical Services, Vanderbilt University Medical Center, 1211 Medical Center Drive, BUH-131, Nashville, TN 37232, USA., joanna.stollings@ vanderbilt.edu.

Data presented, in part, at the Society of Critical Care Medicine 45th Critical Care Congress; Orlando, FL, February 20-24, 2016. Institution where research was performed: Vanderbilt University Medical Center Nashville, Tennessee.

Declaration of Conflicting Interests

The authors declared the following potential conflicts of interest with respect to the research, authorship, and/or publication of this article: Joanna L. Stollings, Sarah L. Bloom, Li Wang, and James Jackson have no conflicts of interest to disclose; E. Wesley Ely has received honoraria for CME activities from Pfizer, Abbott, and Orion and grant support from Dr Franz Kohler Chemie GmbH; Carla M. Sevin has no conflicts of interest to disclose.
} 
Conclusions-Use of a critical care pharmacist resulted in the identification and treatment of multiple medication-related problems in an ICU-RC as well as implementation of preventive measures.

\section{Keywords}

post-intensive care syndrome; post-intensive care syndrome clinic; pharmacist; medication reconciliation; adverse drug event

\section{Introduction}

Post-intensive care syndrome (PICS) refers to new or worsening impairment in mental health, physical function, and cognition following critical illness. ${ }^{1,2}$ Following intensive care unit (ICU) admission, patients with PICS have an increased rate of mortality and are caught in a cycle of recurrent illness and readmissions to the hospital. ${ }^{3}$ Many of these patients do not ever achieve their goal of full recovery. ${ }^{4-8}$ An ICU recovery center (ICU$\mathrm{RC}$ ), also known as a PICS or ICU follow-up clinic, is one potential method of improving long-term care outcomes following critical illness. ${ }^{9-13}$ The goals of an ICU-RC include improvement of cognitive, physical, and psychological function following critical illness, optimized care coordination, and decreased health care use. Nurse-led clinics have been utilized in Europe since the 1990s. ${ }^{11}$ Although the ideal model for an ICU-RC has not been determined, there is a trend toward staffing follow-up clinics with a team that mimics the interprofessional make-up found within many ICUs in the United States. ${ }^{9}$

In 2001, the Society of Critical Care Medicine recognized critical care pharmacists as an integral part of an ICU team. ${ }^{14}$ Critical care pharmacy services have been shown to decrease medication costs ${ }^{15-18}$ and adverse events ${ }^{19-22}$; improve infectious disease-associated morbidity, mortality, and $\operatorname{costs}^{23}$; contribute to better thromboembolic and infarction-related event clinical and economic outcomes ${ }^{24}$; promote appropriate use of stress ulcer prophylaxis ${ }^{25}$; and enhance compliance with ICU protocols such as the ABCDEF (ie,

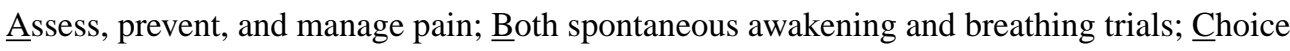
of analgesia and sedation; Delirium-assess, prevent, and manage; Early mobility and exercise; and Family engagement and empowerment) Bundle. ${ }^{26-33}$

Patients are at significant risk for adverse drug events (ADEs) during transitions of care such as discharge from the ICU to the ward or discharge from the ward to home or a facility. ${ }^{34}$ Unnecessary medications are often continued following hospital discharge. ${ }^{25}, 35-45$ Additionally, home maintenance medications are often not resumed on hospital admission and/or subsequent discharge, increasing the risk of death, emergency department visit, or hospitalization. ${ }^{46}$ Given the demonstrated benefit of a critical care pharmacist in the ICU, a critical care pharmacist was integrated into the ICU-RC at Vanderbilt in an attempt to identify and treat the types of medication errors found in a population of high-risk ICU survivors. The objective of this article is to describe the role of an ICU-RC critical care pharmacist in identifying and treating medication-related problems among ICU survivors. 


\section{Methods}

\section{Study Design and Participants}

A prospective, observational cohort study was conducted of all adults referred to the ICU$\mathrm{RC}$ at an academic tertiary care center (Vanderbilt University Medical Center [VUMC]) between July 2012 and December 2015. Patients eligible for follow-up in the ICU-RC were critically ill adults with one or more of the following risk factors for the development of PICS: sepsis/septic shock, delirium, and respiratory failure requiring mechanical ventilation. Exclusion criteria were terminal illness, with life expectancy $<6$ months, hospice referral or comfort care measures only, preexisting cognitive impairment (eg, dementia), residence at a long-term skilled nursing facility, disease-specific follow-up already in place (eg, transplant recipients), and established care with another intensivist (eg, pulmonary physician).

Although medication reconciliation of home medications has been shown to be beneficial in patients with dementia, ${ }^{47}$ patients with cognitive impairment were excluded. The purpose of clinic is to evaluate new cognitive impairment following critical illness.

Patients who met the aforementioned criteria were asked to schedule follow-up with the ICU-RC. Referrals that did not result in an appointment being scheduled were not consistently tracked prior to January 2014. Given the volume of referrals following the first year and the addition of more personnel to the team, a formalized referral-tracking system was adopted. The institutional review board at VUMC approved this study with waiver of informed consent.

\section{The Pharmacist's Role at the ICU-RC Visit}

The ICU-RC comprises an interprofessional team that included a critical care pharmacist, acute care nurse practitioner, pulmonary intensivist, case manager, and neuropsychologist. Each member of the interprofessional team saw the patients individually followed by an interprofessional team conference to develop and execute a comprehensive treatment plan for the patient's primary care provider to ensure optimal transition to outpatient care. The patient's primary care provider was contacted via phone or letter to communicate identified problems, discuss the patient's plan, and to ask or answer any detected questions.

A full medication review, including medication reconciliation, interview, and counseling, was completed and is described in Table 1 . The full medication review was done using a standardized list of questions designed by the pharmacist to assess for indication, appropriate dosage, compliance, and adverse effects of prescription and nonprescription medications. Compliance was assessed for in addition to the patient's vaccination status. Additionally, means to increase medication compliance (eg, use of an alarm clock or changing medications to extended- or sustained-release products) and enhance medication efficacy (eg, counseling on administration time, food/drug interaction, sleep hygiene, and diabetes mellitus) were reviewed where appropriate. The patients were instructed to bring their medications to the clinic. The patient's outpatient pharmacy was only called if the patient did not know what medications he or she was taking or if compliance was in question. 
Drug interactions were recorded. However, to prevent duplication in intervention reporting, they were captured as an ADE or drug therapy discontinued. ADE preventive measures were instituted when a patient was thought to be at risk for an ADE. For example, if a patient was utilizing an inhaled corticosteroid, the patient was potentially at risk for thrush if he or she did not rinse out the mouth after inhaling this medication. If the patient reported not rinsing the month out after utilizing the inhaled corticosteroid, the pharmacist would educate on why this practice is important. All the ADEs were classified by the pharmacist. ADEs were classified as clinically insignificant if unlikely to cause harm. ADEs were classified as significant if the ADE could potentially cause harm and may need further monitoring. If the ADE could potentially cause harm that would likely require further intervention and could result in increased length of hospital stay, the ADE was classified as serious. If the error could lead to death or would require life-sustaining interventions to prevent death, the ADE was classified as life threatening. ${ }^{48,49}$ The pharmacist-identified medication-related problems were then shared with the interprofessional ICU-RC team.

\section{Statistical Analysis}

Categorical data are reported as frequencies and percentages, and continuous data are reported as medians (interquartile ranges). Statistical analyses were performed with $\mathrm{R}$ software version 3.3.0 (R Development Core Team, Vienna, Austria; http:// www.rproject.org).

\section{Results}

Evaluation of 307 patients resulted in 218 referrals to the ICU-RC, with 62 patients completing a visit to the ICU-RC. Of the 62 patients who completed a visit to the ICU-RC, 56 had a full medication review performed by the clinical pharmacist (Figure 1). The triage process is described in Figure 1.

Baseline demographics of patients who were referred to the ICU-RC as compared to those who attended the ICU-RC who were seen by the pharmacist are presented in Table 2 . The median age of patients was 48 years (interquartile range $[\mathrm{IQR}]=35,57$ ). The predominant race was Caucasian. The 3 most common criteria resulting in referrals were respiratory failure requiring mechanical ventilation, delirium, and sepsis/septic shock. The median Sequential Organ Failure Assessment score at ICU admission was $10(\mathrm{IQR}=7,12)$, which correlates with an estimated mortality risk of at least $40 \% .{ }^{50,51}$ Common past medical history among the patients seen in the ICU-RC included the following, listed as $\mathrm{n}(\%)$ : hypertension 21 (38\%); chronic obstructive pulmonary disease 13 (23\%); depression 13 (23\%); diabetes mellitus, 13 (23\%); hyperlipidemia, 13 (23\%); pain, 10 (18\%); gastroesophageal reflux disease, 8 (14\%); renal failure, 7 (13\%); asthma, $6(11 \%)$; hypothyroidism, $6(11 \%)$; coronary artery disease, $6(11 \%)$; congestive heart failure, 6 (11\%); anxiety, $6(11 \%)$; and asthma, $6(11 \%)$.

The majority of patients were enrolled from a 34-bed medical ICU with around 4000 admissions a year. ICU and hospital length of stay were 8 days (IQR $=4,22)$ and 18 days $(\mathrm{IQR}=12,29)$, respectively. The majority of patients were discharged home (without home- 
health services) or to inpatient rehabilitation. The median time from hospital discharge to ICU-RC appointment was 29 days (IQR $=18.57)$.

The median number of pharmacy interventions per patient was $4(\mathrm{IQR}=2,5)$. All 56 patients had at least 1 pharmacy intervention. The number of pharmacy interventions made were per a single visit to the ICU-RC.

The median number of medications the patient was prescribed at the start of the patient visit was $8(\mathrm{IQR}=4,11)$. Medications were stopped in $22(39 \%)$ patients at the clinic appointment. One medication was discontinued in $13(23 \%)$ patients, whereas 2 medications were discontinued in $5(9 \%)$ patients, and 3 medications were discontinued in $2(4 \%)$ patients. A total of 29 medications were discontinued at the ICU-RC (the classes of these medications are listed in Figure 2). New medications were started in 18 (32\%) patients. One medication was initiated in $11(20 \%)$ patients, whereas 2 medications were initiated in 4 (7\%) patients, and 3 medications were initiated in 1 (2\%) patient. In all, 22 new medications were initiated at the ICU-RC; the classes of these medications are listed in Figure 3. The median number of medications the patient was prescribed at the end of the ICU-RC visit was $8(\mathrm{IQR}=4,11)$. Medications were started and stopped in different patients. Overall, the delta change, the difference between the numbers of medications stopped and started, in medications during the clinic visit was $0(\mathrm{IQR}=0,0)$.

An ADE was identified in $9(16 \%)$ patients. The pharmacist identified $8(14 \%)$ patients who had 1 ADE, whereas 1 (2\%) patient had 2 ADEs identified. Two of the ADEs that were treated were considered serious (constipation and oversedation), and 1 was life threatening (hypoglycemia). Multiple drug classes were involved in the ADEs (Table 3). ADE preventive measures were instituted in $18(32 \%)$ patients. One ADE preventive measure was instituted in $16(29 \%)$ patients, whereas $2 \mathrm{ADE}$ preventive measures were initiated in $1(2 \%)$ patient, and $3 \mathrm{ADE}$ preventive measures were instituted in $1(2 \%)$ patient. If these ADEs had occurred, 3 would have been considered significant, 10 classified as serious, and 2 classified as life threatening. Preventive measures initiated by the pharmacist against ADEs included multiple drug classes (Table 3).

A dosage of medication was increased in $9(16 \%)$ patients, whereas $2(4 \%)$ patients had a dosage of a medication decreased. Drug classes that were increased and decreased are listed in Table 4. Refills were written in $2(4 \%)$ patients.

A laboratory test related to a medication was ordered in $6(11 \%)$ patients. The individual laboratory tests are listed in Table 4. A medication organizer was provided to $8(14 \%)$ patients. Interventions used to increase compliance occurred 29 times, whereas interventions to increase efficacy occurred 77 times. In all, 17 (30\%) patients received blood pressure teaching (Table 4). An influenza vaccination was administered to 13 (23\%) patients, and pneumococcal vaccination was administered to $2(4 \%)$ patients.

\section{Discussion}

In this study, investigating the contributions of an ICU-RC critical care pharmacist in an outpatient ICU-RC setting, we demonstrated that significant medication-related management 
was warranted in this high-risk ICU survivor population. Medication errors and risks were consistently addressed and eliminated in the early post-ICU period, often before patients saw their primary physician. This is a descriptive study in which each of these medically complex patients had up to 30 minutes of subspecialty medication reconciliation that they would not have received in routine care. The ICU-RC team's familiarity with the patient's ICU course, having treated them in the ICU, allowed the team to make medication decisions where someone unfamiliar with the ICU course or indication for the medications prescribed therein may not have been able to do. The results presented here describe how the utilization of a critical care pharmacist in an ICU-RC may facilitate identification and treatment of multiple medication problems as well as the standardization of preventive measures such as vaccines following critical illness.

The findings of this study are consistent with prior studies demonstrating the continuation of unnecessary medications following critical illness. ${ }^{25,} 35-40,42-44$ In a single-center study of 120 elderly, adult patients following critical illness, 250 potentially inappropriate medications (PIMs) were prescribed at discharge. In all, 36\% of the PIMS were considered to be actually inappropriate medications (AIMs). Almost two-thirds of AIMs were initiated in the ICU. ${ }^{35}$ Continuation of antipsychotics initiated in the ICU at hospital discharge has been shown in multiple other studies. ${ }^{36-40}$ Several studies have documented the inappropriate continuation of acid-suppressive therapy at hospital discharge following an ICU stay. ${ }^{25,}, 42-44$

The results of this study align with previous studies that demonstrate the importance of continuing chronic medications. A large cohort study of 396380 elderly Canadians evaluated outpatient and hospital medication prescriptions. In this study, patients admitted to the hospital were more likely to have appropriate home medications discontinued compared with nonhospitalized patients. ${ }^{46}$ In patients hospitalized with an ICU stay, the risk of medication discontinuation was higher when compared with patients hospitalized without an ICU stay. Patients who had a statin or antiplatelet agent discontinued had an increased composite risk of death, hospitalization, and emergency department visits for up to 1 year following hospital discharge (odds ratio $=1.07 ; 95 \% \mathrm{CI}=1.03-1.11) .{ }^{46}$

Although many critically ill patients have overt immunosuppression, numerous other critically ill patients have conditions that impair immunity, such as chronic alcoholism, malnourishment, renal insufficiency, and liver failure. Regardless of underlying immunocompromising comorbidities, a significant proportion of critically ill patients can be classified as immunocompromised through clinical and laboratory findings such as loss of body temperature control with or without new-onset hypothermia, hypoalbuminemia in spite of sufficient nutritional support, continuing low absolute lymphocyte counts, inability to treat the infection despite source control and appropriate antibiotics, and development of opportunistic infections and ICU-acquired infections. ${ }^{52}$ Immunizations are withheld in critically ill patients during the ICU stay, yet they can and often should be given prior to hospital discharge. If a live vaccination is given to an immunocompromised patient, the patient could become infected. A fever associated with a vaccination may be mistaken for an infection. In patients with primary or secondary immunodeficiency, an adequate immune response may not be able to be mounted against an inactivated vaccination. ${ }^{53}$ Despite the 
risk factors for influenza in this population, many patients did not receive timely vaccination prior to hospital discharge. In this study, many patients needed and received influenza vaccine in the clinic, despite inpatient protocols to ensure such vaccination prior to hospital discharge.

As the ICU-RC team members all work in the medical ICU, a majority of the patients seen in the ICU-RC were referred from there. Patients in our medical ICU setting are complex and appear to benefit from specialized follow-up; however, critically ill patients from other ICUs are not excluded. The ICU-RC is actively working to expand services to patients from other ICUs.

It is unknown whether this degree of medication management would have been accomplished by other members of the ICU-RC without the addition of a critical care pharmacist. When considering whether or not to deploy a critical care pharmacist to work in the ICU-RC, many factors need to be considered. The pharmacist's familiarity with the patient and ICU population is an obvious benefit. Pharmacist-conducted medication histories, when compared with those of other health care practitioners, have been shown to have a lower rate of errors. ${ }^{54,55}$ In many cases, the medication reconciliation performed by the pharmacist in the ICU-RC is a continuation of care provided in the ICU. If the same pharmacist who works in the ICU works in the ICU-RC, the pharmacist would be absent from the ICU while working in the clinic. However, with an established pharmacist, the ICU team could contact the pharmacist with needs while the pharmacist works in the ICU-RC. The ICU-RC in this study requires approximately 3 hours bimonthly, which results in minimal pharmacist absence from the ICU.

In many states, pharmacists cannot bill for medication management services because they are not officially recognized as providers. Additionally, in states where pharmacists are recognized as providers, billing is often not supported by the institution because reimbursement rates for pharmacists are low and, therefore, not worth the additional administrative burden. The inability to bill could potentially result in a pharmacist balancing a heavy work load in the ICU and the ICU-RC. In many institutions, a pharmacist not only performs clinical activities but is responsible for processing medication orders for patients in the ICU. If the pharmacist was working in the ICU-RC, another individual would need to be responsible for medication order processing during that time frame.

Although an ICU-RC has the potential to reduce morbidity associated with PICS, improve long-term outcomes, and decrease readmission rates to the hospital, there are limitations to establishing and maintaining an ICU-RC. Basic resources needed to identify and treat problems associated with critical illness include a physical clinic location with laboratory monitoring capabilities, a team of interprofessional providers, and a process for recruiting patients.

No specific protocols have been developed concerning evaluation of the indication or duration of thrombotic or gastric prophylaxis or medications used for treatment of delirium at the time of discharge from the ICU. However, the critical care pharmacist ensures the discontinuation of these medications in patients who no longer have an indication. 
Unfortunately, these medications are sometimes started by the ward team and continued on discharge. Although some patients do receive discharge medication reconciliation, VUMC does not have a formalized discharge medication reconciliation process. Even if a formalized medication discharge process did exist, $17 \%$ of patients were discharged to a skilled nursing facility, and $7 \%$ of patients were discharged to long-term acute care. New medications could have been started at these facilities. It is unknown if the same outcomes would have been obtained with improvements in the discharge process.

In addition, we have observed in the ICU-RC that orders and medication changes made at the time of discharge are often not carried out or are incorrectly carried out. This observation emphasizes that the early posthospital period is a high-risk transition time that, in complex patients, requires closer follow-up. This is a call for improved medication reconciliation at all transitions of care (admission, transfer to ICU, transfer to ward, and discharge).

This study has several strengths. We describe the potential function of a pharmacist in an ICU-RC, which has never been previously described. This cohort study helps fill a gap in the literature by describing the potential role of including a critical care pharmacist in the interprofessional team caring for patients after critical illness. The results of this study echo numerous studies demonstrating the ability of a critical care pharmacist to identify and treat medication-related problems in the ICU, but little is known regarding the medication needs and potential harms to patients once they leave the hospital. Many of the medication-related problems appear to be related to the ICU stay. ${ }^{35-43,56}$ The patients in this cohort were seen by the same pharmacist in and after the ICU, providing a continuity of care not usually found between the ICU and the posthospital period.

This study also has limitations that should be recognized. The cohort studied was at a single academic medical center, which might limit extrapolation of results. No comparison group of similar patients who were not seen by the pharmacist at the ICU-RC was readily available. However, this is the first article to describe the role of a critical care pharmacist at an ICU-RC. When indicated, the ICU-RC physician or nurse practitioner prescribes medications and immunizations, makes specialty referrals, and orders durable medical equipment. As health insurance coverage is fragmented in the United States, the case manager, usually a nurse, coordinates care coverage and links the patients with resources to help cover the costs of necessary care, equipment, and medications.

However, although many medications were stopped at the ICU-RC, it is unknown if the patient's primary care provider restarted these medications. It has been hypothesized in multiple studies that clinicians fear discontinuing patients' medications because of lack of clarity regarding the indication. ${ }^{41,57}$ The delta change in medications during the clinic visit was 0 (IQR $=0,0)$, which seems counterintuitive given the multiple medication adjustments. Medications were discontinued and alternative medications were started in multiple cases. For example, in one patient, a proton pump inhibitor was discontinued in favor of an asneeded histamine 2 blocker to treat the patient's intermittent gastroesophageal reflux disease while minimizing the risk of numerous complications associated with proton pump inhibitors. ${ }^{56,58-61}$ 


\section{Conclusions}

In conclusion, a critical care pharmacist visit in the context of an ICU-RC resulted in the identification and treatment of multiple medication-related problems as well as the implementation of preventive measures, which may or may not have prevented ADEs from occurring. Much remains to be learned about medication risks and needs in the post-ICU population. This article serves as a call for action to improve transitions of care following both ICU and hospital discharge. Although the ideal model of an ICU-RC is not known, the results of this report suggest an expanded role for a critical care pharmacist because a member of an ICU-RC interprofessional team could potentially benefit patients and minimize adverse outcomes following critical illness. Further studies could be directed toward evaluating long-term patient outcomes, multiple patient/pharmacist visits, the pharmacist/family interaction, and the pharmacoeconomic impact of the ICU-RC pharmacist's interventions.

\section{Acknowledgments}

Funding

The authors disclosed receipt of the following financial support for the research, authorship, and/or publication of this article: The study received financial support from the Vanderbilt Institute for Clinical and Translational Research (VR21637, VR11449, VR7052), the Vanderbilt Division of Allergy, Pulmonary and Critical Care Medicine, and the Vanderbilt Department of Pharmaceutical Services. Dr Ely has been supported by the VA Clinical Science Research and Development Service (Washington, DC) and the National Institutes of Health AG027472, AG035117, and HL111111 (Bethesda, MD).

\section{References}

1. Needham DM, Davidson J, Cohen H, et al. Improving long-term outcomes after discharge from intensive care unit: report from a stakeholders' conference. Crit Care Med. 2012; 40:502-509. [PubMed: 21946660]

2. Elliott D, Davidson JE, Harvey MA, et al. Exploring the scope of post-intensive care syndrome therapy and care: engagement of non-critical care providers and survivors in a second stakeholders meeting. Crit Care Med. 2014; 42:2518-2526. DOI: 10.1097/CCM.0000000000000525 [PubMed: 25083984]

3. Unroe M, Kahn JM, Carson SS, et al. One-year trajectories of care and resource utilization for recipients of prolonged mechanical ventilation: a cohort study. Ann Intern Med. 2010; 153:167-175. [PubMed: 20679561]

4. Herridge MS, Cheung AM, Tansey CM, et al. One-year outcomes in survivors of the acute respiratory distress syndrome. N Engl J Med. 2003; 348:683-693. DOI: 10.1056/NEJMoa022450 [PubMed: 12594312]

5. Jackson JC, Hart RP, Gordon SM, et al. Six-month neuropsychological outcome of medical intensive care unit patients. Crit Care Med. 2003; 31:1226-1234. [PubMed: 12682497]

6. Iwashyna TJ, Ely EW, Smith DM, et al. Long-term cognitive impairment and functional disability among survivors of severe sepsis. JAMA. 2010; 304:1787-1794. DOI: 10.1001/jama.2010.1553 [PubMed: 20978258]

7. Davydow DS. Symptoms of depression and anxiety after delirium. Psychosomatics. 2009; 50:309_ 316. DOI: 10.1176/appi.psy.50.4.309 [PubMed: 19687169]

8. Wunsch H, Christiansen CF, Johansen MB, et al. Psychiatric diagnoses and psychoactive medication use among nonsurgical critically ill patients receiving mechanical ventilation. JAMA. 2014; 311:1133-1142. DOI: 10.1001/jama.2014.2137 [PubMed: 24643603] 
9. Huggins EL, Bloom SL, Stollings JL, et al. A clinic model: post-intensive care syndrome and postintensive care syndrome-family. AACN Adv Crit Care. 2016; 27:204-211. DOI: 10.4037/ aacnacc2016611 [PubMed: 27153309]

10. Stollings JL, Caylor MM. Postintensive care syndrome and the role of a follow-up clinic. Am J Health Syst Pharm. 2015; 72:1315-1323. DOI: 10.2146/ajhp140533 [PubMed: 26195658]

11. Griffiths JA, Barber VS, Cuthbertson BH, et al. A national survey of intensive care follow-up clinics. Anaesthesia. 2006; 61:950-955. DOI: 10.1111/j.1365-2044.2006.04792.x [PubMed: 16978309]

12. Cuthbertson BH, Rattray J, Campbell MK, et al. The PRaCTICaL study of nurse led, intensive care follow-up programmes for improving long term outcomes from critical illness: a pragmatic randomised controlled trial. BMJ. 2009; 339:b3723.doi: 10.1136/bmj.b3723 [PubMed: 19837741]

13. Jensen JF, Egerod I, Bestle MH, et al. A recovery program to improve quality of life, sense of coherence and psychological health in ICU survivors: a multicenter randomized controlled trial, the RAPIT study. Intensive Care Med. 2016; 42:1733-1743. DOI: 10.1007/s00134-016-4522-1 [PubMed: 27695894]

14. Brilli RJ, Spevetz A, Branson RD, et al. Critical care delivery in the intensive care unit: defining clinical roles and the best practice model. Crit Care Med. 2001; 29:2007-2019. [PubMed: 11588472]

15. Montazeri M, Cook DJ. Impact of a clinical pharmacist in a multidisciplinary intensive care unit. Crit Care Med. 1994; 22:1044-1048. [PubMed: 8205814]

16. Weant KA, Armitstead JA, Ladha AM, et al. Cost effectiveness of a clinical pharmacist on a neurosurgical team. Neurosurgery. 2009; 65:946-950. discussion 950-951. DOI: 10.1227/01.NEU. 0000347090.22818.35 [PubMed: 19834408]

17. Kane SL, Weber RJ, Dasta JF. The impact of critical care pharmacists on enhancing patient outcomes. Intensive Care Med. 2003; 29:691-698. DOI: 10.1007/s00134-003-1705-3 [PubMed: 12665997]

18. Wu JY, Stollings JL, Wheeler AP, Semler MW, Rice TW. Efficacy and outcomes after vasopressin guideline implementation in septic shock. Ann Pharmacother. 2017; 51:13-20. DOI: 10.1177/1060028016669163 [PubMed: 27630192]

19. Leape LL, Cullen DJ, Clapp MD, et al. Pharmacist participation on physician rounds and adverse drug events in the intensive care unit. JAMA. 1999; 282:267-270. [PubMed: 10422996]

20. Ng TM, Bell AM, Hong C, et al. Pharmacist monitoring of QTc interval-prolonging medications in critically ill medical patients: a pilot study. Ann Pharmacother. 2008; 42:475-482. DOI: 10.1345/ aph.1K458 [PubMed: 18319393]

21. Rivkin A, Yin H. Evaluation of the role of the critical care pharmacist in identifying and avoiding or minimizing significant drug-drug interactions in medical intensive care patients. J Crit Care. 2011; 26:104-e6. e1-e6. DOI: 10.1016/j.jcrc.2010.04.014

22. Hamblin S, Rumbaugh K, Miller R. Prevention of adverse drug events and cost savings associated with PharmD interventions in an academic level I trauma center: an evidence-based approach. J Trauma Acute Care Surg. 2012; 73:1484-1490. DOI: 10.1097/TA.0b013e318267cd80 [PubMed: 23064610]

23. MacLaren R, Bond CA, Martin SJ, et al. Clinical and economic outcomes of involving pharmacists in the direct care of critically ill patients with infections. Crit Care Med. 2008; 36:3184-3189. DOI: 10.1097/CCM.0b013e31818f2269 [PubMed: 18936700]

24. MacLaren R, Bond CA. Effects of pharmacist participation in intensive care units on clinical and economic outcomes of critically ill patients with thromboembolic or infarction-related events. Pharmacotherapy. 2009; 29:761-768. DOI: 10.1592/phco.29.7.761 [PubMed: 19558249]

25. Tasaka CL, Burg C, VanOsdol SJ, et al. An interprofessional approach to reducing the overutilization of stress ulcer prophylaxis in adult medical and surgical intensive care units. Ann Pharmacother. 2014; 48:462-469. DOI: 10.1177/1060028013517088 [PubMed: 24473490]

26. Marshall J, Finn CA, Theodore AC. Impact of a clinical pharmacist-enforced intensive care unit sedation protocol on duration of mechanical ventilation and hospital stay. Crit Care Med. 2008; 36:427-433. [PubMed: 18091554] 
27. Stollings JL, Foss JJ, Ely EW, et al. Pharmacist leadership in ICU quality improvement: coordinating spontaneous awakening and breathing trials. Ann Pharmacother. 2015; 49:883-891. DOI: 10.1177/1060028015582050 [PubMed: 25907528]

28. MacLaren R, Plamondon JM, Ramsay KB, et al. A prospective evaluation of empiric versus protocol-based sedation and analgesia. Pharmacotherapy. 2000; 20:662-672. [PubMed: 10853622]

29. Devlin JW, Marquis F, Riker RR, et al. Combined didactic and scenario-based education improves the ability of intensive care unit staff to recognize delirium at the bedside. Crit Care. 2008; 12:R19.doi: 10.1186/cc6793 [PubMed: 18291021]

30. Gesin G, Russell BB, Lin AP, et al. Impact of a delirium screening tool and multifaceted education on nurses' knowledge of delirium and ability to evaluate it correctly. Am J Crit Care. 2012; 21:e1e11. DOI: 10.4037/ajcc2012605 [PubMed: 22210704]

31. Swan JT. Decreasing inappropriate unable-to-assess ratings for the confusion assessment method for the intensive care unit. Am J Crit Care. 2014; 23:60-69. DOI: 10.4037/ajcc2014567 [PubMed: 24382618]

32. Louzon P, Jennings H, Ali M, et al. Impact of pharmacist management of pain, agitation, and delirium in the intensive care unit through participation in multidisciplinary bundle rounds. Am J Health Syst Pharm. 2017; 74:253-262. DOI: 10.2146/ajhp150942 [PubMed: 28179250]

33. Marino J, Bucher D, Beach M, et al. Implementation of an intensive care unit delirium protocol: an interdisciplinary quality improvement project. Dimens Crit Care Nurs. 2015; 34:273-284. DOI: 10.1097/DCC.0000000000000130 [PubMed: 26244242]

34. Hume AL, Kirwin J, et al. American College of Clinical Pharmacy. Improving care transitions: current practice and future opportunities for pharmacists. Pharmacotherapy. 2012; 32:e326-e337. DOI: 10.1002/phar.1215 [PubMed: 23108810]

35. Morandi A, Vasilevskis E, Pandharipande PP, et al. Inappropriate medication prescriptions in elderly adults surviving an intensive care unit hospitalization. J Am Geriatr Soc. 2013; 61:11281134. DOI: 10.1111/jgs.12329 [PubMed: 23855843]

36. Tomichek JE, Stollings JL, Pandharipande PP, et al. Antipsychotic prescribing patterns during and after critical illness: a prospective cohort study. Crit Care. 2016; 20:378.doi: 10.1186/ s13054-016-1557-1 [PubMed: 27881149]

37. Kram BL, Kram SJ, Brooks KR. Implications of atypical anti-psychotic prescribing in the intensive care unit. J Crit Care. 2015; 30:814-818. DOI: 10.1016/j.jcrc.2015.03.030 [PubMed: 25887805]

38. Flurie RW, Gonzales JP, Tata AL, et al. Hospital delirium treatment: continuation of antipsychotic therapy from the intensive care unit to discharge. Am J Health Syst Pharm. 2015; 72(23, suppl 3):S133-S139. DOI: 10.2146/ajhp150474 [PubMed: 26582298]

39. Jasiak KD, Middleton EA, Camamo JM, et al. Evaluation of discontinuation of atypical antipsychotics prescribed for ICU delirium. J Pharm Pract. 2013; 26:253-256. DOI: 10.1177/0897190012465987 [PubMed: 23184410]

40. Rowe AS, Hamilton LA, Curtis RA, et al. Risk factors for discharge on a new antipsychotic medication after admission to an intensive care unit. J Crit Care. 2015; 30:1283-1286. DOI: 10.1016/j.jcrc.2015.08.009 [PubMed: 26383104]

41. McDonald EG, Jones J, Green L, et al. Reduction of inappropriate exit prescriptions for proton pump inhibitors: a before-after study using education paired with a web-based qualityimprovement tool. J Hosp Med. 2015; 10:281-286. DOI: 10.1002/jhm.2330 [PubMed: 25708942]

42. Murphy CE, Stevens AM, Ferrentino N, et al. Frequency of inappropriate continuation of acid suppressive therapy after discharge in patients who began therapy in the surgical intensive care unit. Pharmacotherapy. 2008; 28:968-976. DOI: 10.1592/phco.28.8.968 [PubMed: 18657013]

43. Pavlov A, Muravyev R, Amoateng-Adjepong Y, et al. Inappropriate discharge on bronchodilators and acid-blocking medications after ICU admission: importance of medication reconciliation. Respir Care. 2014; 59:1524-1529. DOI: 10.4187/respcare.02913 [PubMed: 24847095]

44. Shin S. Evaluation of costs accrued through inadvertent continuation of hospital-initiated proton pump inhibitor therapy for stress ulcer prophylaxis beyond hospital discharge: a retrospective chart review. Ther Clin Risk Manag. 2015; 11:649-657. DOI: 10.2147/TCRM.S81759 [PubMed: 26005351] 
45. George C, Verghese J. Polypharmacy and gait performance in community-dwelling older adults. J Am Geriatr Soc. 2017; 65:2082-2087. DOI: 10.1111/jgs.14957 [PubMed: 28649786]

46. Bell CM, Brener SS, Gunraj N, et al. Association of ICU or hospital admission with unintentional discontinuation of medications for chronic diseases. JAMA. 2011; 306:840-847. DOI: 10.1001/ jama.2011.1206 [PubMed: 21862745]

47. Pfister B, Jonsson J, Gustafsson M. Drug-related problems and medication reviews among old people with dementia. BMC Pharmacol Toxicol. 2017; 18:52.doi: 10.1186/s40360-017-0157-2 [PubMed: 28655357]

48. Classen DC, Pestotnik SL, Evans RS, et al. Adverse drug events in hospitalized patients: excess length of stay, extra costs, and attributable mortality. JAMA. 1997; 277:301-306. [PubMed: 9002492]

49. Folli HL, Poole RL, Benitz WE, et al. Medication error prevention by clinical pharmacists in two children's hospitals. Pediatrics. 1987; 79:718-722. [PubMed: 3575028]

50. Ferreira FL, Bota DP, Bross A, et al. Serial evaluation of the SOFA score to predict outcome in critically ill patients. JAMA. 2001; 286:1754-1758. [PubMed: 11594901]

51. Vincent JL, Mendonca Ad, Cantraine F, et al. Use of the SOFA score to assess the incidence of organ dysfunction/failure in intensive care units: results of a multicenter, prospective study. Crit Care Med. 1998; 26:1793-1800. [PubMed: 9824069]

52. Pene F, Pickkers P, Hotchkiss RS. Is this critically ill patient immunocompromised? Intensive Care Med. 2016; 42:1051-1054. DOI: 10.1007/s00134-015-4161-y [PubMed: 26630877]

53. Kroger, AT., Duchin, J., Vázquez, M. [Accessed February 2, 2018] General best practice guidelines for immunization: best practices of the Advisory Committee on Immunization Practices (ACIP)http://www.cdc.gov/vaccines/hcp/acip-recs/general-recs/downloads/general-recs.pdf

54. Reeder TA, Mutnick A. Pharmacist- versus physician-obtained medication histories. Am J Health Syst Pharm. 2008; 65:857-860. DOI: 10.2146/ajhp070292 [PubMed: 18436732]

55. Pippins JR, Gandhi TK, Hamann C, et al. Classifying and predicting errors of inpatient medication reconciliation. J Gen Intern Med. 2008; 23:1414-1422. DOI: 10.1007/s11606-008-0687-9 [PubMed: 18563493]

56. Barletta JF, Sclar DA. Proton pump inhibitors increase the risk for hospital-acquired Clostridium difficile infection in critically ill patients. Crit Care. 2014; 18:714.doi: 10.1186/ s13054-014-0714-7 [PubMed: 25540023]

57. Koczka CP, Geraldino-Pardilla LB, Goodman AJ. Physicians' opinions of stress ulcer prophylaxis: survey results from a large urban medical center. Dig Dis Sci. 2013; 58:777-781. DOI: 10.1007/ s10620-012-2423-x [PubMed: 23065088]

58. Miano TA, Reichert MG, Houle TT, et al. Nosocomial pneumonia risk and stress ulcer prophylaxis: a comparison of pantoprazole vs ranitidine in cardiothoracic surgery patients. Chest. 2009; 136:440-447. DOI: 10.1378/chest.08-1634 [PubMed: 19318661]

59. Khalili H, Huang ES, Jacobson BC, et al. Use of proton pump inhibitors and risk of hip fracture in relation to dietary and lifestyle factors: a prospective cohort study. BMJ. 2012; 344:e372.doi: 10.1136/bmj.e372 [PubMed: 22294756]

60. Gomm W, von Holt K, Thome F, et al. Association of proton pump inhibitors with risk of dementia: a pharmacoepidemiological claims data analysis. JAMA Neurol. 2016; 73:410-416. DOI: 10.1001/jamaneurol.2015.4791 [PubMed: 26882076]

61. Lazarus B, Chen Y, Wilson FP, et al. Proton pump inhibitor use and the risk of chronic kidney disease. JAMA Intern Med. 2016; 176:238-246. DOI: 10.1001/jamainternmed.2015.7193 [PubMed: 26752337] 


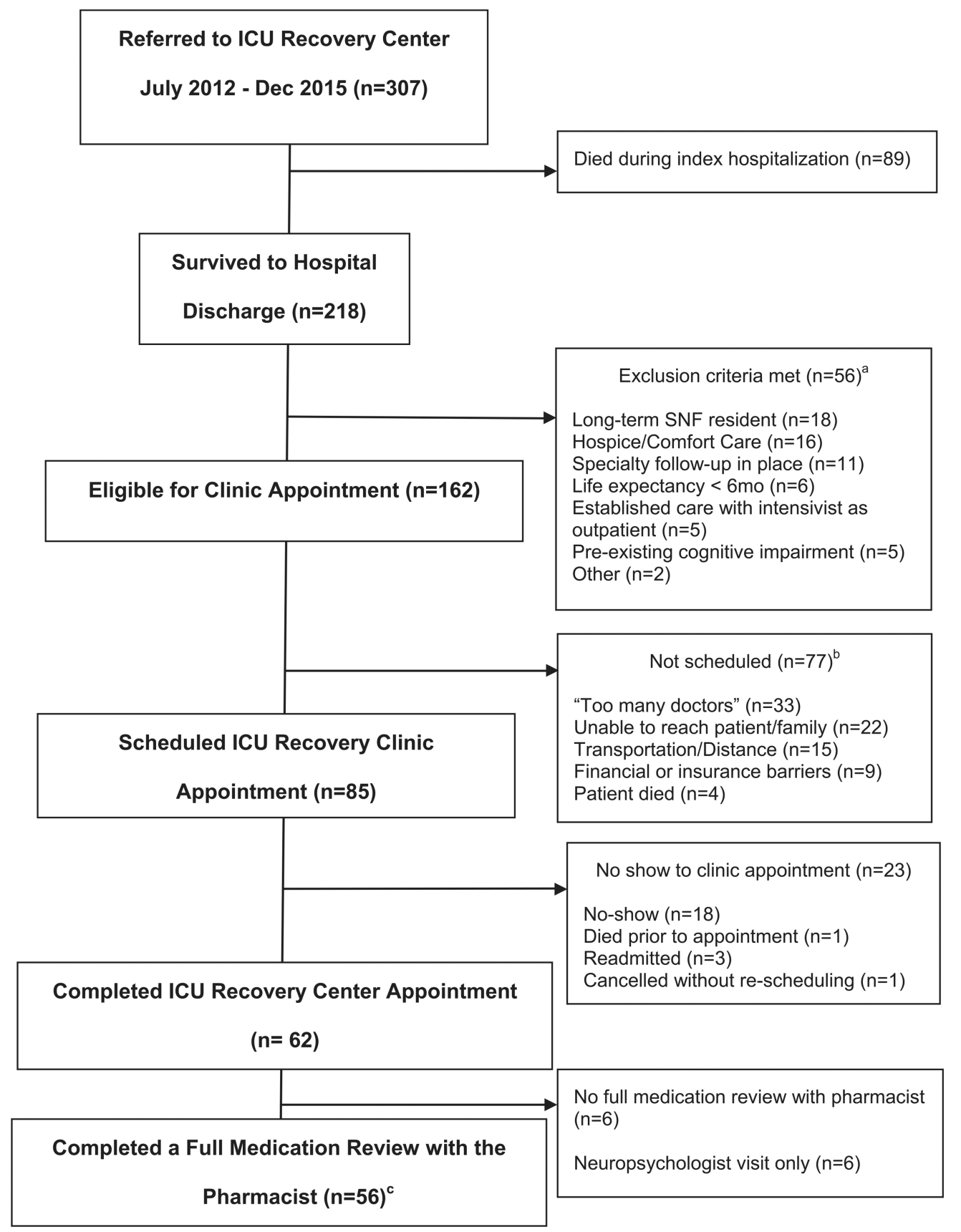

Figure 1. Flow diagram of patients seen by the pharmacist at the intensive care unit recovery center

Abbreviations: ICU-RC, intensive care unit recovery center; SNF, skilled nursing facility; mo, months. 


\section{Medications Discontinued at the ICU-RC}

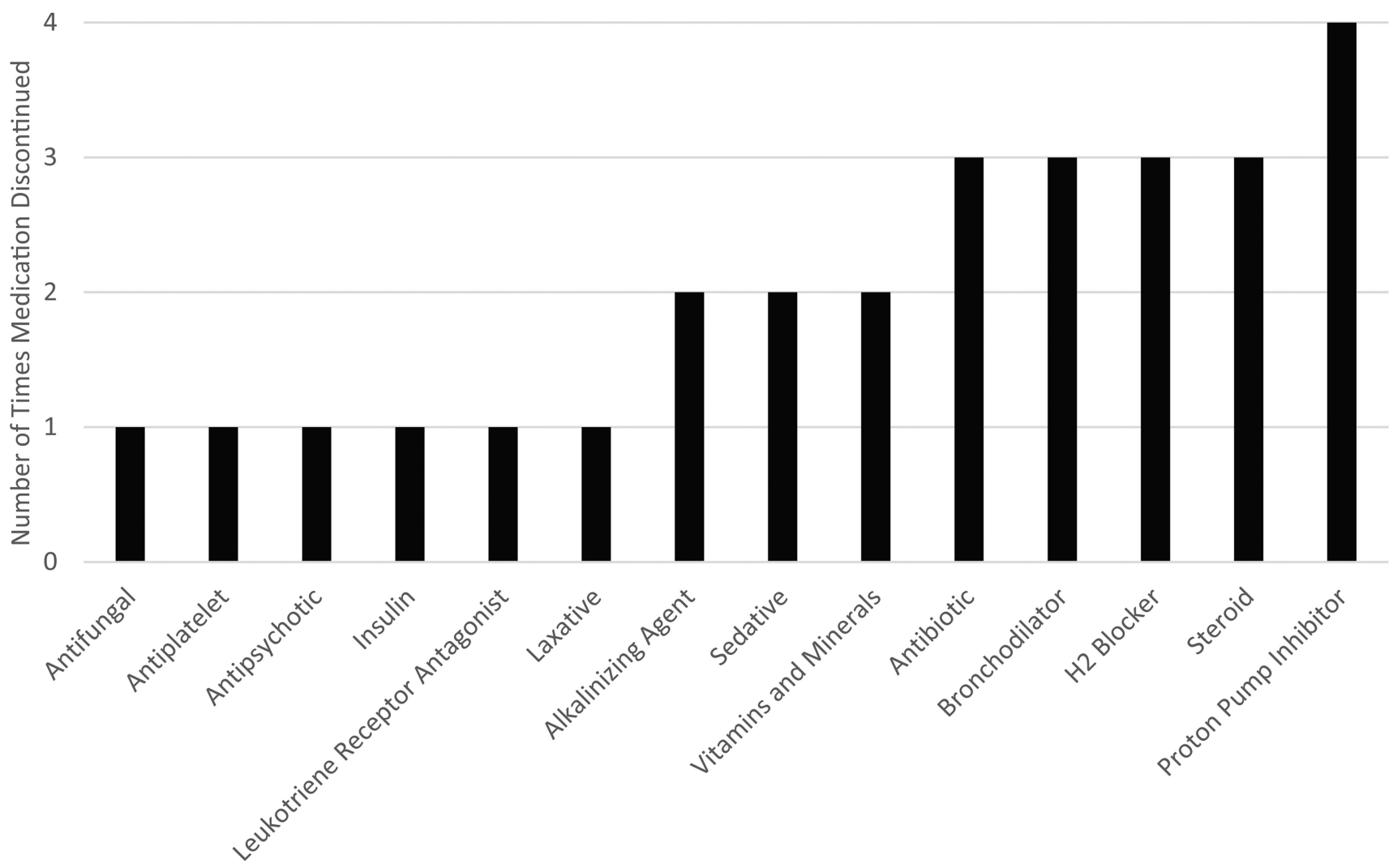

Figure 2. Medications discontinued at the intensive care unit recovery center Abbreviations: $\mathrm{H} 2$, histamine 2; ICU-RC, intensive care unit recovery center. 


\section{Medications Started at the ICU-RC}

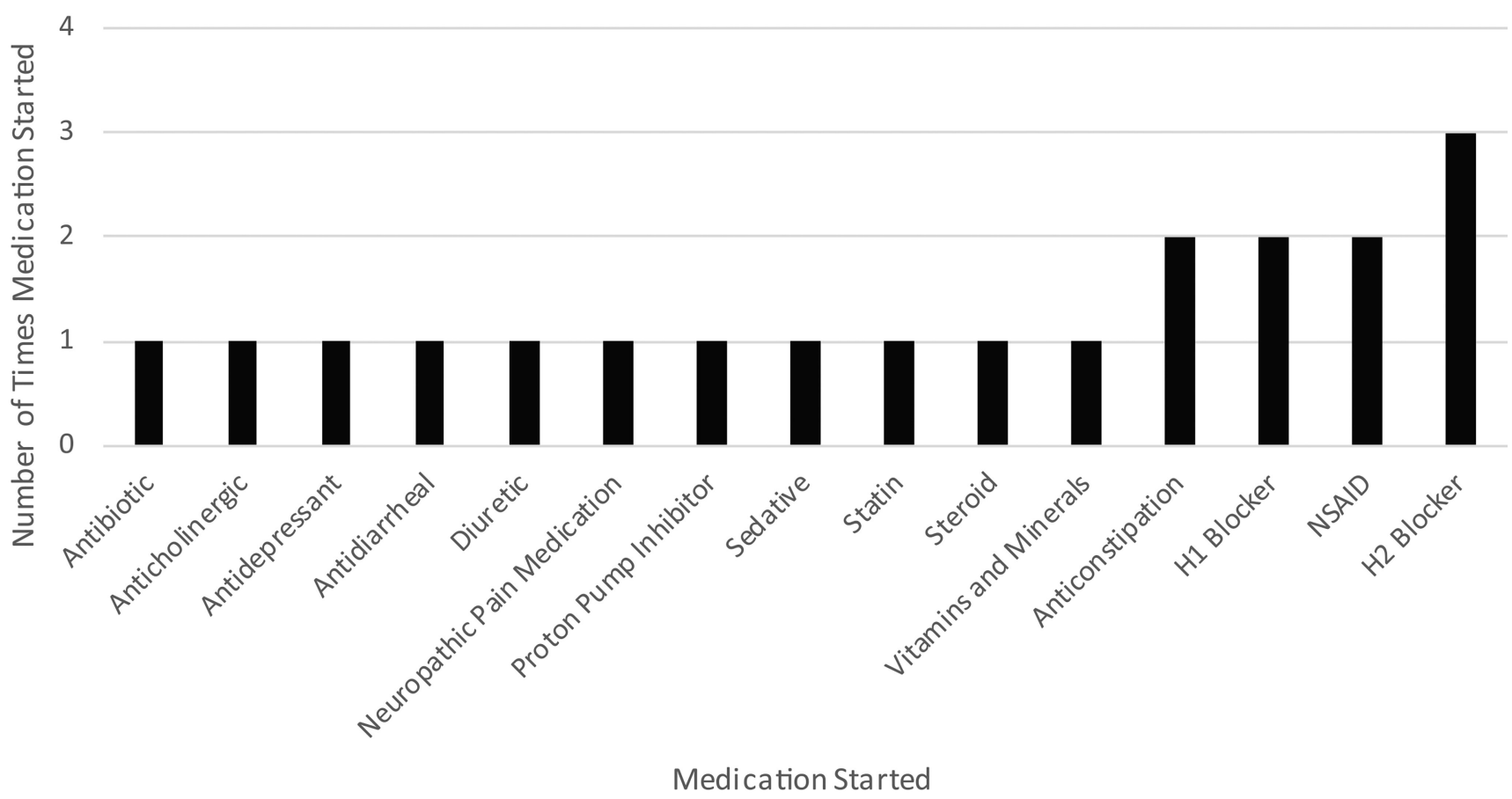

Figure 3. Medications started at the intensive care unit recovery center

Abbreviations: $\mathrm{H} 1$, histamine 1; $\mathrm{H} 2$, histamine 2; ICU-RC, intensive care unit recovery center; NSAID, nonsteroidal anti-inflammatory drug. 


\title{
Table 1
}

\section{Medication-Related Questions Asked at the Intensive Care Unit Recovery Center.}

\author{
1. What is the indication for each medication? \\ 2. What is the dose and frequency for each medication? \\ 3. How does the patient take each medication (ie, what time of day, with meals, etc)? \\ 4. How often does the patient miss a dose? What does he/she do if they miss a dose? \\ 5. Does the patient use a medication organizer? \\ 6. What side effects has the patient experienced with the medication? \\ 7. How much and how often does the patient take their prn medications? \\ 8. Does the patient take any over-the-counter medications? \\ 9. Does the patient take any herbal medications? \\ 10. Does the patient visit one pharmacy? \\ 11. Does the patient know to ask the physician or pharmacist prior to taking any over-the-counter or herbal medications? \\ 12. Is the cost of medications a barrier to obtaining medications? \\ 13. Does the patient smoke? \\ 14. Does the patient drink alcohol? \\ 15. Does the patient use illicit drugs? \\ 16. Does the patient take their blood pressure at home? \\ 17. Does the patient have regular bowel movements? \\ 18. Does the patient meet criteria for an influenza vaccine? \\ 19. Does the patient meet criteria for a pneumococcal vaccine? \\ 20 . Does the patient need refills of any medications?
}

Abbreviations: prn, pro re nata (when necessary). 
Table 2

Baseline Demographics of Survivors Who Were Referred Versus Attended the ICU-RC. ${ }^{a}$

\begin{tabular}{|c|c|c|}
\hline Demographic & $\begin{array}{c}\text { Survivors } \\
\text { Referred } \\
(\mathbf{n}=\mathbf{2 1 8})^{b}\end{array}$ & $\begin{array}{l}\text { Survivors } \\
\text { Attended } \\
(\mathrm{n}=56)^{c}\end{array}$ \\
\hline Age, years & $55(43,65)$ & $48(35,57)$ \\
\hline Sex, male & $105(48 \%)$ & $32(57 \%)$ \\
\hline Race, white & $177(81 \%)$ & $48(86 \%)$ \\
\hline SOFA score & $10(7,12)$ & $10(7,12)$ \\
\hline \multicolumn{3}{|l|}{ Referral ICU type } \\
\hline Medical ICU & $197(90 \%)$ & $49(88 \%)$ \\
\hline Trauma & $7(3 \%)$ & $3(5 \%)$ \\
\hline CVICU & $6(3 \%)$ & $2(4 \%)$ \\
\hline Surgical ICU & $4(2 \%)$ & \\
\hline Other & $4(2 \%)$ & $2(4 \%)$ \\
\hline \multicolumn{3}{|l|}{ ICU-RC referral criteria ${ }^{d}$} \\
\hline Respiratory failure & $166(76 \%)$ & $47(84 \%)$ \\
\hline Delirium during ICU stay & $162(74 \%)$ & $45(80 \%)$ \\
\hline Sepsis/Septic shock & $147(67 \%)$ & $31(55 \%)$ \\
\hline Shock, other & $88(40 \%)$ & $15(27 \%)$ \\
\hline ICU length of stay, days & $8(4,14.8)$ & $8(4,22)$ \\
\hline Hospital length of stay, days & $15(9.2,25.8)$ & $18(12,29)$ \\
\hline \multicolumn{3}{|l|}{ Discharge disposition } \\
\hline Home with self-care & $57(26 \%)$ & $19(34 \%)$ \\
\hline Home with home health & $41(19 \%)$ & $10(18 \%)$ \\
\hline Inpatient rehabilitation & $41(19 \%)$ & $16(29 \%)$ \\
\hline Skilled nursing facility & $38(17 \%)$ & $5(9 \%)$ \\
\hline Long-term acute care & $16(7 \%)$ & $5(9 \%)$ \\
\hline Home with hospice & $11(5 \%)$ & \\
\hline Left against medical advice & $5(2 \%)$ & $1(2 \%)$ \\
\hline Inpatient hospice & $3(1 \%)$ & \\
\hline Inpatient psychiatric hospital & $2(1 \%)$ & \\
\hline Other & $4(2 \%)$ & \\
\hline
\end{tabular}

Abbreviations: CVICU, cardiovascular intensive care unit; ICU, intensive care unit; ICU-RC, intensive care unit recovery center; SOFA, Sequential Organ Failure Assessment.

${ }^{a}$ Data presented as $\mathrm{n}(\%)$ or median (interquartile range).

$b_{\text {Includes all survivors referred to the ICU-RC. }}$

Includes only survivors who attended the ICU-RC and were seen by a pharmacist.

${ }^{d}$ Referral criteria were not mutually exclusive. 
Table 3

Drug Class and Corresponding Adverse Drug Events in the ICU-RC. ${ }^{a}$

\begin{tabular}{lll}
\hline $\begin{array}{l}\text { Drug Class Involved in ADE } \\
\text { Experienced (n= 10) }\end{array}$ & $\mathbf{n = 5 6}$ & ADE Experienced \\
\hline$\beta$-Blockers & $1(2 \%)$ & Fatigue \\
Insulin & $1(2 \%)$ & Hypoglycemia \\
Leukotriene receptor antagonists & $1(2 \%)$ & Hallucinations \\
Opiates & $1(2 \%)$ & Constipation \\
Sedatives & $1(2 \%)$ & Oversedation \\
Antibiotics & $2(4 \%)$ & Emesis, nausea \\
Steroids & $3(5 \%)$ & Insomnia, peripheral edema, thrush \\
\hline Drug Class Involved in ADE Prevention Measures Initiated $(\mathrm{n}=21)$ & & ADE Preventive Measures Initiated Against \\
\hline Analgesics (nonopiates) & $1(2 \%)$ & Overdose \\
Antiplatelet & $1(2 \%)$ & Thrombosis \\
Bronchodilators & $1(2 \%)$ & Overdose \\
Decongestants & $1(2 \%)$ & Overdose \\
Oral diabetes medications & $1(2 \%)$ & Hypoglycemia \\
Sedatives & $2(4 \%)$ & Dizziness $(\mathrm{n}=1)$, oversedation $(\mathrm{n}=1)$ \\
Antibiotics & $3(5 \%)$ & Nausea $(\mathrm{n}=2)$, photosensitivity $(\mathrm{n}=1)$ \\
Proton pump inhibitors & $3(5 \%)$ & Osteoporosis \\
Opiates & $4(8 \%)$ & Constipation $(\mathrm{n}=3)$, overdose $(\mathrm{n}=1)$ \\
Steroids & $4(8 \%)$ & Thrush \\
\hline
\end{tabular}

Abbreviations: ADE, adverse drug event; ICU-RC, intensive care unit recovery center

${ }^{a}$ Data presented as n (\%). 
Table 4

Miscellaneous Pharmacy Interventions Performed Through the ICU-RC. ${ }^{a}$

\begin{tabular}{ll}
\hline & $\mathbf{n = 5 6}$ \\
\hline Dose of medication increased & \\
Neuropathic pain medications & $4(8 \%)$ \\
Anticoagulants & $1(2 \%)$ \\
Antidepressants & $1(2 \%)$ \\
Anxiolytics & $1(2 \%)$ \\
Expectorants & $1(2 \%)$ \\
Opiates & $1(2 \%)$ \\
Dose of medication decreased & \\
Anticoagulants & $1(2 \%)$ \\
Opiates & $1(2 \%)$ \\
Refills written & \\
Bronchodilators & $1(2 \%)$ \\
Steroids & $1(2 \%)$ \\
Laboratory test ordered & \\
Basic metabolic panel & $1(2 \%)$ \\
Potassium & $1(2 \%)$ \\
Liver function tests & $1(2 \%)$ \\
Complete blood count & $1(2 \%)$ \\
Thyroid stimulating hormone & $1(2 \%)$ \\
Thyroxine & $1(2 \%)$ \\
\hline
\end{tabular}

Abbreviation: ICU-RC, intensive care unit recovery center.

a Data presented as n (\%). 